\title{
COALESCENCE OF SKEW BROWNIAN MOTIONS
}

\section{Martin Barlow, ${ }^{1}$ Krzysztof Burdzy, ${ }^{2}$ Haya Kaspi ${ }^{3}$ and Avi Mandelbaum ${ }^{3}$}

The purpose of this short note is to prove almost sure coalescence of two skew Brownian motions starting from different initial points, assuming that they are driven by the same Brownian motion. The result is very simple but we would like to record it in print as it has already become the foundation of a research project of Burdzy and Chen (1999). Our theorem is a by-product of an investigation of variably skewed Brownian motion, see Barlow et al. (1999).

Suppose that $B_{t}$ is the standard Brownian motion with $B_{0}=0$ and consider the equation

$$
X_{t}^{x}=x+B_{t}+\beta L_{t}^{x}, \quad t \geq 0,
$$

where $X_{t}^{x}$ satisfies the initial condition $X_{0}^{x}=x$. Here $\beta$ is a fixed number in $[-1,1]$ and $L_{t}^{x}$ is the symmetric local time of $X_{t}^{x}$ at 0 . Harrison and Shepp (1981) proved that (1) has a unique strong solution, which is skew Brownian motion. One way to define skew Brownian motion in the case $\beta \geq 0$ is to start with a standard Brownian motion $B_{t}^{\prime}$ and flip every excursion of $B_{t}^{\prime}$ below 0 to the positive side with probability $\beta$, independent of what happens to other excursions. See Itô and McKean (1965) or Walsh (1978) for more information.

Theorem. If $X_{t}^{x}$ and $X_{t}^{y}$ are solutions of (1) with the same $\beta \in[-1,1] \backslash\{0\}$, relative to the same Brownian motion $B_{t}$, then $X_{t}^{x}=X_{t}^{y}$ for some $t<\infty$, a.s.

Proof. For simplicity assume that $\beta>0$ and $0=x<y$. Let $\widehat{L}_{t}^{0}=\beta L_{t}^{0}, \widehat{L}_{t}^{y}=y+\beta L_{t}^{y}$,

1. Research partially supported by an NSERC (Canada) grant.

2. Research partially supported by NSF grant DMS-9700721.

3. Research partially supported by the Fund for the Promotion of Research at the Technion. 
and

$$
\begin{aligned}
T_{0}= & 0, \\
S_{k}= & \inf \left\{t>T_{k}:-B_{t}=\widehat{L}_{t}^{y}\right\}, \quad k \geq 0, \\
T_{k}= & \inf \left\{t>S_{k-1}:-B_{t}=\widehat{L}_{t}^{0}\right\}, \quad k \geq 1, \\
W_{k}= & \frac{\widehat{L}_{S_{k-1}}^{y}-\widehat{L}_{S_{k-1}}^{0}}{\widehat{L}_{T_{k-1}}^{y}-\widehat{L}_{T_{k-1}}^{0}}, \quad k \geq 1, \\
V_{k}= & \frac{\widehat{L}_{T_{k}}^{y}-\widehat{L}_{T_{k}}^{0}}{\widehat{L}_{S_{k-1}}^{y}-\widehat{L}_{S_{k-1}}^{0}}, \quad k \geq 1, \\
M_{k}= & \widehat{L}_{T_{k}}^{y}-\widehat{L}_{T_{k}}^{0}, \quad k \geq 0 .
\end{aligned}
$$

We will first find the distributions of $W_{k}$ 's and $V_{k}$ 's using excursion theory. Recall the fundamentals of excursion theory for the standard Brownian motion from, e.g., Karatzas and Shreve (1991). The Brownian excursions from 0 form a Poisson point process whose clock can be identified with the local time of Brownian motion at 0 . The intensity of excursions on the positive side of 0 whose height is greater than $h$ is equal to $1 /(2 h)$.

The stopping time $S_{0}$ may be described as the first time when an excursion of $-B_{t}-\widehat{L}_{t}^{0}$ above 0 hits the level $y-\widehat{L}_{t}^{0}$. These excursions can be identified with the excursions of the skew Brownian motion $X_{t}^{0}$ below 0. They form a Poisson point process $\mathcal{P}$ similar to the Poisson point process of excursions of the standard Brownian motion from 0. The intensity of $\mathcal{P}$-excursions above 0 with height greater than $h$ is equal to $(1-\beta) /(2 h)$. Note the extra factor $1-\beta$ as compared to the analogous formula for the excursions of the standard Brownian motion. The factor can be explained using the excursion flipping construction of skew Brownian motion mentioned in the introduction - in a sense, the fraction of excursions flipped to the other side is equal to $\beta / 2$. When the clock $L_{t}^{0}$ for the Poisson point process $\mathcal{P}$ takes a value $u$ then the instanteneous intensity of excursions with height greater than $y-\widehat{L}_{t}^{0}$ is equal to $(1-\beta) /(2(y-\beta u))$. We have $\widehat{L}_{S_{0}}^{y}-\widehat{L}_{S_{0}}^{0}<a$ if no $\mathcal{P}$-excursion with height greater than $y-\widehat{L}_{t}^{0}$ occurs before the time $s$ when $y-\widehat{L}_{s}^{0}=a$, i.e., when $L_{s}^{0}=(y-a) / \beta$. Thus excursion theory enables us to write the probability of this event using Poisson probabilities as follows,

$$
P\left(\widehat{L}_{S_{0}}^{y}-\widehat{L}_{S_{0}}^{0}<a\right)=\exp \left(-\int_{0}^{(y-a) / \beta} \frac{1-\beta}{2(y-\beta u)} d u\right)=\left(\frac{a}{y}\right)^{(1-\beta) /(2 \beta)}
$$

Recall that $\widehat{L}_{T_{0}}^{y}-\widehat{L}_{T_{0}}^{0}=y$. We have

$$
P\left(W_{1} y<a\right)=P\left(W_{1}\left(\widehat{L}_{T_{0}}^{y}-\widehat{L}_{T_{0}}^{0}\right)<a\right)=P\left(\widehat{L}_{S_{0}}^{y}-\widehat{L}_{S_{0}}^{0}<a\right)=(a / y)^{(1-\beta) /(2 \beta)}
$$


By changing the variable we obtain for $w \in(0,1)$,

$$
P\left(W_{1}<w\right)=w^{(1-\beta) /(2 \beta)} .
$$

By the strong Markov property, $P\left(W_{k}<w\right)=w^{(1-\beta) /(2 \beta)}$ for $w \in(0,1)$ and every $k \geq 1$.

A totally analogous argument shows that $P\left(V_{k}>v\right)=v^{-(1+\beta) /(2 \beta)}$ for $v \geq 1$ and $k \geq 1$.

Note that, by the strong Markov property, all random variables $V_{k}, W_{k}, k \geq 1$, are jointly independent.

Next we will show that the process $M_{k}$ is a martingale and converges to 0 . First, note that $M_{k}=M_{k-1} W_{k} V_{k}$. It is elementary to check that $E W_{k}=(1-\beta) /(1+\beta)$ and $E V_{k}=(1+\beta) /(1-\beta)$. By the joint independence of $W_{k}$ 's and $V_{k}$ 's,

$$
E\left(M_{k} \mid M_{k-1}, M_{k-2}, \ldots\right)=M_{k-1} E W_{k} E V_{k}=M_{k-1},
$$

which shows that $M_{k}$ is a martingale. As a positive martingale, the process $M_{k}$ must converge with probability 1 to a random variable $M_{\infty}$. Since for every $k, M_{k}$ is the product of $M_{k-1}$ and an independent random variable $W_{k} V_{k}$, the limit $M_{\infty}$ can take only the values 0 or $\infty$. By Fatou's Lemma, $E M_{\infty} \leq E M_{0}=y$, so $M_{\infty}=0$ a.s.

On every interval $\left[T_{k}, S_{k}\right]$ the process $\widehat{L}_{t}^{y}-\widehat{L}_{t}^{0}$ is non-increasing but it is non-decreasing on intervals of the form $\left[S_{k}, T_{k+1}\right]$. Thus

$$
\sup _{t \in\left[T_{k}, T_{k+1}\right]} \widehat{L}_{t}^{y}-\widehat{L}_{t}^{0} \leq \max \left(M_{k}, M_{k+1}\right) .
$$

In view of convergence of $M_{k}$ to 0 , we must have a.s. convergence of $\widehat{L}_{t}^{y}-\widehat{L}_{t}^{0}$ to 0 when $t \rightarrow \infty$. It remains to show that the convergence does not take an infinite amount of time.

Let $T_{\infty}=\lim _{k \rightarrow \infty} T_{k}$. In view of the remarks in the last paragraph, it is not hard to see that the value of $\widehat{L}_{T_{\infty}}^{0}$ is bounded by $\sum_{k=1}^{\infty} M_{k}$. Since $\widehat{L}_{\infty}^{0}=\infty$, it will suffice to show that $\sum_{k=1}^{\infty} M_{k}<\infty$ in order to conclude that $T_{\infty}<\infty$. We have for $k \geq 1$,

$$
M_{k}=y \prod_{j=1}^{k} W_{j} V_{j}
$$

We can write

$$
y \prod_{j=1}^{k} W_{j} V_{j}=\exp \left(\log y+\sum_{j=1}^{k}\left[\log W_{j}+\log V_{j}\right]\right) .
$$


One can directly check that the distribution of $-\log W_{j}$ is exponential with mean $2 \beta /(1-$ $\beta$ ), while the distribution of $\log V_{j}$ is exponential with mean $2 \beta /(1+\beta)$. Thus, $E\left(\log W_{j}+\right.$ $\left.\log V_{j+1}\right)<0$. It follows that for some $a>0$, we eventually have

$$
\sum_{j=1}^{k}\left[\log W_{j}+\log V_{j}\right] \leq-a k
$$

Hence, for some random $c_{1}$ and all $k$ we have $M_{k} \leq c_{1} e^{-a k}$ and so $\sum_{k=1}^{\infty} M_{k}<\infty$, a.s.

\section{References}

[1] Barlow, M., Burdzy, K., Kaspi, H. and Mandelbaum, A. (1999), Variably skewed Brownian motion (preprint)

[2] Burdzy, K. and Chen, Z.-Q. (1999) Local time flow related to skew Brownian motion (preprint)

[3] Harrison, J.M. and Shepp, L.A. (1981), On skew Brownian motion, Ann. Probab. 9 (2), 309-313.

[4] Itô, K. and McKean, H.P. (1965), Diffusion Processes and Their Sample Paths, Springer, New York.

[5] Karatzas, I. and Shreve, S.E. (1991), Brownian Motion and Stochastic Calculus, 2nd Edition, Springer Verlag, New York.

[6] Walsh, J.B. (1978), A diffusion with discontinuous local time, Temps Locaux Asterisque, 52-53, 37-45.

Martin Barlow: University of British Columbia, Vancouver, BC V6T 1Z2, Canada barlow@math.ubc.ca

Krzysztof Burdzy: University of Washington, Seattle, WA 98195-4350, USA burdzy@math.washington.edu

Haya Kaspi and Avi Mandelbaum: Technion Institute, Haifa, 32000, Israel iehaya@tx.technion.ac.il,avim@tx.technion.ac.il 